\title{
Theoretical Study of the Interplay of Superconductivity and Magnetism in FeAs Based Superconductors
}

\author{
Mesfin A. Afrassa ${ }^{1}$, Poran Singh ${ }^{2}$ \\ Department of Physics, Addis Ababa University, Addis Ababa, Ethiopia \\ Email: mes my@yahoo.com, psinghgbpub@yahoo.com
}

Received 25 January 2014; revised 27 February 2014; accepted 11 March 2014

Copyright $@ 2014$ by authors and Scientific Research Publishing Inc.

This work is licensed under the Creative Commons Attribution International License (CC BY).

http://creativecommons.org/licenses/by/4.0/

c) (i) Open Access

\begin{abstract}
The interaction of superconductivity and magnetism is studied in iron based superconductor using the Hamiltonian consisting of the itinerant electrons, localized electrons moment, and s-f interaction. Using Greens function technique and equation of motion method, we have obtained an expressions for superconducting order parameters $(\Delta(T), \Delta(0))$ and critical temperature $T_{C}$, which reduce to BCS result in the absence of magnetic interactions. The result of the calculations shows that superconductivity can coexist with magnetism in iron based superconductor below the critical temperature.
\end{abstract}

Keywords

Superconductivity, Magnetism, Coexistence, Green's Function

\section{Introduction}

The discovery of high $\mathrm{T}_{\mathrm{C}}$ iron based superconductor in 2008 [1] boosts multidirectional investigation from experimental as well as theoretical views. Nowadays, understanding the mechanism of superconductivity in such system is one of the challenging research areas. According to reviews on iron based superconductors [2] [3], magnetic interactions are important for understanding the mechanism of superconductivity. Experimental observation and theoretical prediction show that knowing the interplay of superconductivity and magnetism may suggest the possible mechanism of superconductivity.

The interplay of superconductivity and magnetism has been studied in iron based superconductors theoretically and experimentally [4] [5]. Superconductivity could be obtained applying either external pressure or dop-

How to cite this paper: Afrassa, M.A. and Singh, P. (2014) Theoretical Study of the Interplay of Superconductivity and Magnetism in FeAs Based Superconductors. World Journal of Condensed Matter Physics, 4, 53-57.

http://dx.doi.org/10.4236/wjcmp.2014.42008 
ing. In most iron based superconductors, both electron and hole doping on parent compounds cause superconductivity. Upon doping, for example, potassium doping magnetism gradually disappears with a lowering of the spin density wave transition temperature [6]. In systems like $\mathrm{CeFeAsO}_{1-x} \mathrm{~F}_{x}$, magnetism is suppressed by doping before the appearance of superconductivity [7]. Generally substitution of element in the 122 parent compounds may lead to suppression of spin density wave and eventual appearance of superconductivity [4] [8]. Some compounds show a coexistence of magnetism and superconductivity [9]-[11]

In this work, we are trying to predict the interplay of superconductivity and magnetism on iron based superconductors which can help in explaining experimental observations.

\section{The Model Hamiltonian}

Our model Hamiltonian is composed of

$$
H=H_{P}+H_{l}+H_{e l},
$$

where the first term, $H_{p}=\sum_{k, \sigma} \varepsilon(k) \hat{a}_{k \sigma}^{\dagger} \hat{a}_{k \sigma}-\sum_{k k^{\prime}} V_{k k^{\prime}} \hat{a}_{k \uparrow}^{\dagger} \hat{a}_{-k \downarrow}^{\dagger} \hat{a}_{-k \downarrow} \hat{a}_{-k^{\prime} \downarrow} \hat{a}_{k^{\prime} \uparrow}$.

In the above pairing Hamiltonian the term

$$
\sum_{k, \sigma} \varepsilon(k) \hat{a}_{k \sigma}^{\dagger} \hat{a}_{k \sigma},
$$

describes the Hamiltonian of total energy of the itinerant electrons in one electron band approximation [12]. Here the operators $\hat{a}_{k \sigma}^{\dagger}\left(\hat{a}_{k \sigma}\right)$ creates (annihilates) an electron with the wave vector $\mathrm{k}$ and the spin projection on z-axis $\sigma=\uparrow$ or $\downarrow ; V_{k k^{\prime}}$ is the BCS pair potential. The second term $\left(H_{l}=\sum_{i, j} J S_{i} \cdot S_{j}\right)$ describes the predominant interaction between the local moment by Heisenberg like model, and we considered only the nearest neighbor interaction. Here $J$ is the nearest neighbor exchange that bridge by the As ions and it could be anti ferromagnetic in nature. The third term $\left(H_{e l}=-g \sum_{i} \sigma_{i} \cdot S_{i}\right)$ describes the interaction between the spin $\sigma_{i}$ of the itinerant electrons and the five $3 \mathrm{~d}$ spin $S_{i}$ local moment located at site $i$, where $g$ is the corresponding exchange constant.

To get an effective interaction we change the momentum term in to boson operator. Diagonalizing the Hamiltonian $\left(\mathrm{H}_{1}\right)$ using Bogoliubov transformation, the canonical form of the Hamiltonian in terms of spin waves,

$$
H_{l}=E_{O}+\sum_{k} \omega_{k} b_{k}^{\dagger} b_{k}
$$

We obtained the itinerant electrons and localized electrons moment using relations in spin operators like,

$$
S_{i}^{\sigma}=\hat{a}_{i \sigma}^{\dagger} \hat{a}_{i-\sigma}, \frac{1}{\hbar} \sigma_{i}^{\dagger}=\hat{a}_{i \downarrow}^{\dagger} \hat{a}_{i \uparrow}, \frac{1}{\hbar} \sigma_{i}^{z}=\frac{1}{2} \sum_{\sigma} z_{\sigma} n_{i \sigma} ;\left(z_{\uparrow}=+1 ; z_{\downarrow}=-1\right) .
$$

The electrons in the valence band which are interacting with an anti ferromagnetically ordered, localized spin system can be described by

$$
H_{e l}=-\frac{1}{2} g\left\langle S^{z}\right\rangle \sum_{k, \sigma} \hat{a}_{k \sigma}^{\dagger} \hat{a}_{k \sigma}
$$

We get an effective Hamiltonian

$$
H_{\text {eff }}=\sum_{k, \sigma} \varepsilon(k) \hat{a}_{k \sigma}^{\dagger} \hat{a}_{k \sigma}-\sum_{k k^{\prime}} V_{k k^{\prime}} \hat{a}_{k \uparrow}^{\dagger} \hat{a}_{-k \downarrow}^{\dagger} \hat{a}_{-k \downarrow} \hat{a}_{-k^{\prime} \downarrow} \hat{a}_{k^{\prime} \uparrow}+E_{O}+\sum_{k} \omega_{k} \hat{b}_{k}^{\dagger} \hat{b}_{k}-\frac{1}{2} g \hbar\left\langle S^{z}\right\rangle \sum_{k \sigma} \hat{a}_{k \sigma}^{\dagger} \hat{a}_{k \sigma}
$$

In order to calculate the superconducting parameter, we first need to obtain equation of motion. In this work we used Greens function equation of motion method. Applying elementary commutation relation we found two equations:

$$
\begin{array}{r}
(\omega-\varepsilon)\left\langle\left\langle a_{-k \downarrow}^{\dagger}, a_{k^{\prime} \uparrow}^{\dagger}\right\rangle\right\rangle>>=-\Delta\left\langle\left\langle a_{k^{\prime} \uparrow}, a_{k^{\prime} \uparrow}^{\dagger}\right\rangle\right\rangle \\
(\omega+\varepsilon)\left\langle\left\langle a_{k^{\prime} \uparrow}, a_{k^{\prime} \uparrow}^{\dagger}\right\rangle\right\rangle=1-\Delta\left\langle\left\langle a_{-k \downarrow}^{\dagger}, a_{k^{\prime} \uparrow}^{\dagger}\right\rangle\right\rangle
\end{array}
$$


From these we get,

$$
\left\langle\left\langle a_{-k \downarrow}^{\dagger}, a_{k^{\prime} \uparrow}^{\dagger}\right\rangle\right\rangle=\frac{-\Delta}{\omega^{2}-\varepsilon^{2}-\Delta^{2}}
$$

where $\varepsilon=\varepsilon_{k}-1 / 2\left(g \hbar\left\langle S^{z}\right\rangle\right)$ and $\langle\langle\ldots\rangle\rangle$ is the abbreviated notation for the Green functions.

The superconducting order parameter can be expressed as

$$
\Delta=\sum_{k, n} \frac{V_{k k^{\prime}}}{\beta}\left\langle\left\langle a_{-k \downarrow}^{\dagger}, a_{k^{\prime} \uparrow}^{\dagger}\right\rangle\right\rangle
$$

The sum may be changed to integral by introducing the density of state $N(\varepsilon)$ and the above equation becomes

$$
1=\frac{1}{\beta} \sum \int \mathrm{d} \varepsilon_{o} N(\varepsilon) V_{k k^{\prime}}\left[\frac{-1}{\omega^{2}-\varepsilon^{2}-\Delta^{2}}\right]
$$

Attractive interaction is effective for the region $-\hbar \omega_{O}<\varepsilon<\hbar \omega_{O}$ and assuming the density of states does not vary over this integral, then the expression becomes,

$$
\frac{1}{\lambda}=\int_{0}^{\hbar \omega_{0}} \frac{2}{\beta} \sum_{0}^{\infty} \frac{-1}{\omega^{2}-\varepsilon^{2}-\Delta^{2}}
$$

Applying Laplaces transform with replacement of $\omega$ by Matsubara frequency $\omega_{n}=(2 n+1) \pi / \beta$, and using the approximation,

$$
\frac{1}{\omega_{n}^{2}+\varepsilon^{2}+\Delta^{2}} \approx \frac{1}{\omega_{n}^{2}+\varepsilon^{2}}-\frac{\Delta^{2}}{\left(\omega_{n}^{2}+\varepsilon^{2}\right)^{2}}
$$

The equation becomes

$$
\frac{1}{\lambda}=\sum_{0}^{\hbar \omega_{0}} \mathrm{~d} \varepsilon \frac{\tanh (\beta / 2) \varepsilon}{\varepsilon}-4 \Delta^{2} \sum_{n=0}^{\infty}\left(\frac{\beta}{\pi(2 n+1)^{2}}\right)^{3} \int_{0}^{\infty} \frac{1}{\left(1+x^{2}\right)^{2}} \mathrm{~d} x
$$

For low temperature the first integral becomes

$$
I_{1}=\ln \frac{1.13}{K_{B} T}\left(\hbar \omega_{0}-\frac{1}{2} g \hbar\left\langle S^{z}\right\rangle\right)
$$

The second integral becomes

$$
I_{2}=1.05\left(\frac{\Delta}{\pi K_{B} T}\right)^{2}
$$

Hence,

$$
\frac{1}{\lambda}=\ln \frac{1.13}{K_{B} T}\left(\hbar \omega_{0}-\frac{1}{2} g \hbar\left\langle S^{z}\right\rangle\right)-1.05\left(\frac{\Delta}{\pi k_{B} T}\right)^{2}
$$

This expression can be rewritten as

$$
K_{B} T=1.13\left(\hbar \omega_{0}-\frac{1}{2} g \hbar\left\langle S^{Z}\right\rangle\right) e^{-\left[\frac{1}{\lambda}+\left(\frac{\Delta}{\pi k_{B} T}\right)^{2}\right]}
$$

\section{Result}

From this equation we can get the following important relations.

1) Superconducting order parameter as a function of temperature 


$$
\Delta(T)=\pi k_{B} T \sqrt{\left(\ln \frac{1.13\left(\hbar \omega_{0}-1 / 2\left(g \hbar\left\langle S^{z}\right\rangle\right)\right)}{k_{B} T}\right)-\frac{1}{\lambda}}
$$

This quantity $(\Delta)$ is zero at critical temperature $T_{C}$. Substituting $\Delta=0$, we get

$$
K_{B} T=1.13\left(\hbar \omega_{0}-\frac{1}{2} g \hbar\left\langle S^{z}\right\rangle\right) \mathrm{e}^{-\frac{1}{\lambda}}
$$

2) using $\ln (1-x)=x-x^{2} / 2+\cdots, \ln T / T_{C} \approx-\left(1-T / T_{C}\right)$ we get the well known equation

$$
\Delta(T)=3.06 k_{B} T_{C}\left(1-\frac{1}{T_{C}}\right)^{2}
$$

Or

$$
\ln \left(T_{C} / T\right)=\left(\frac{\Delta}{\pi k_{B} T}\right)^{2}
$$

3) Equation (9) can be written as

$$
\frac{1}{\lambda}=\sum_{0}^{\hbar \omega_{0}} \mathrm{~d} \varepsilon \frac{\tanh \beta / 2 \sqrt{\varepsilon^{2}+\Delta^{2}}}{\sqrt{\varepsilon^{2}+\Delta^{2}}}
$$

As $T \rightarrow 0$, and $\beta \rightarrow 0$, gives

$$
\frac{1}{\lambda}=\int \frac{\mathrm{d} \varepsilon}{\sqrt{\varepsilon^{2}+\Delta^{2}}}
$$

Applying standard integrals and approximation for $x \ll 1$, and $(1+x)^{n} \approx 1+n x$, we get

$$
\Delta(T \rightarrow 0)=\sqrt{4\left(\hbar \omega_{0}\right)^{2} \exp (-1 / \lambda)-\left(\frac{1}{2} g \hbar\left\langle S^{z}\right\rangle\right)^{2}}
$$

\section{Conclusions}

Equation (13) is clearly in agreement with the fact that as the net magnetization increases, the induction of superconductivity decreases. In addition to this, in the absence of magnetic term, Equation (13) reduces to the well known BCS expression.

The results clearly show that superconductivity can coexist with magnetism in iron based superconductor below the critical temperature. Experimental findings show the coexistence of superconductivity and magnetism in some range of doping in some compounds [9]-[11]. Our theoretical predictions are in broad agreement with experimental findings [9]-[11], and the result may help in explaining experimental observations.

\section{References}

[1] Kamihara, Y., Watanabe, T., Hirano, M. and Hosono, H. (2008) Iron-Based Layered Superconductor La[O1-xFx] FeAs $(\mathrm{x}=0.05-0.12)$ with Tc $=26$ K. Journal of the American Chemical Society, 130, 3296-3297. http://dx.doi.org/10.1021/ja800073m

[2] Lumsden, M.D. and Christianson, A.D. (2010) Magnetism in Fe-Based Superconductors. Condensed Matter Physics, 22, Article ID: 203203.

[3] Dai, P.C., Hu, J.P. and Dagotto, E. (2012) Magnetism and Its Microscopic Origin in Iron-Based High-Temperature Superconductors. http://arxiv.org/abs/1209.0381v1

[4] Ruebenbauer, K., Żukrowski, J., Bukowski, Z., Matusiak, M. and Karpinski, J. (2012) Interplay between Spin Density Wave and Superconductivity in “122” Iron Pnictides: 57Fe Mössbauer Study. Acta Physica Polonica A, 121, 726-729.

[5] Vorontsov, A.B., Vavilov, M. G. and Chubukov, A.V. (2009) Interplay between Magnetism and Superconductivity in 
Fe-Pnictides. Physical Review B, 79, Article ID: 060508 (R). http://dx.doi.org/10.1103/PhysRevB.79.060508

[6] Rotter, M., et al. (2009) Competition of Magnetism and Superconductivity in under Doped (Ba1-xKx) Fe2As2. New Journal of Physics, 11, Article ID: 025014. http://dx.doi.org/10.1088/1367-2630/11/2/025014

[7] Zhao, J., et al. (2008) Structural and Magnetic Phase Diagram of CeFeAsO1-xFx and Its Relation to High-Temperature Superconductivity. Nature Materials, 7, 953-959. http://dx.doi.org/10.1038/nmat2315

[8] Blachowski, A., et al. (2011) Coexistence of Superconductivity and Magnetism Theoretical Predictions and Experimental Result. Physical Review B, 84, Article ID: 174503. http://dx.doi.org/10.1080/00018738500101741

[9] Nandi, N.S., et al. (2010) Anomalous Suppression of the Orthorhombic Lattice Distortion in Superconducting Ba (Fe1xCox) (2) As-2 Single Crystals. Physical Review Letters, 104, Article ID: 057006. http://dx.doi.org/10.1103/PhysRevLett.104.057006

[10] Han, F., Zhu, X.Y., Cheng, P., et al. (2009) Superconductivity and Phase Diagrams of the 4d-and 5d-Metal-Doped Iron Arsenides SrFe2-xMxAs2 (M=Rh,Ir,Pd). Physical Review B, 80, Article ID: 024506. http://dx.doi.org/10.1007/s11433-009-0302-z

[11] Chen, H., et al. (2009) Coexistence of the Spin-Density Wave and Superconductivity in Ba1-xKxFe2As2. Europhysics Letters, 85, Article ID: 17006. http://dx.doi.org/10.1209/0295-5075/85/17006

[12] William, D.B. (2009) Fundamentals of Many-Body Physics Principles and Methods. Springer-Verlag, Berlin Heidelberg. 